\title{
Factors Associated with the Frequency of Doing Domestic Chores After Mild to Moderate Stroke
}

\author{
Kohei Kusuda $^{1}$, Rumi Tanemura ${ }^{2}$ \\ ${ }^{1}$ Kyoto Min-Iren Asukai Hospital \\ ${ }^{2}$ Kobe University
}

\begin{abstract}
Background: While many studies research factors that affect Instrumental Activities of Daily Living after stroke, few studies research factors that affect domestic chores after stroke. This study aims to investigate factors that affect domestic chores after stroke.

Methods: In this cohort study, 29 stroke patients were followed from the time they entered the rehabilitation ward to one month after discharge. Participants were included if they had been independently doing domestic chores before stroke onset and were independently walking inside the hospital after stroke onset. Variables were selected from demographics, physical function, cognitive function, psychological function, and functioning. The Spearman correlation between the domestic chores score of the Frenchay Activities Index (FAI) after stroke and variables was calculated.

Results: The Timed Up and Go test $(r=0.41, p=0.03)$, the Stroke Self-Efficacy Questionnaire $(r=0.54, p<0.01)$ and the Functional Independence Measure motor domain $(r=0.57, p<0.01)$ were significantly associated with the domestic chores domain of the FAI. Unlike previous studies, cognitive function and depression also didn't show a statistical relationship with domestic chores after stroke.

Conclusion: Our results show that stroke patients should improve their self-efficacy to resume domestic chores after stroke, in addition to physical and cognitive functions. The results are also consistent with previous studies about the research relationship between self-efficacy and functioning.
\end{abstract}

Keywords: Stroke, domestic chores, IADL, self-efficacy, balance ability

(Asian J Occup Ther 17: 9-16, 2021)

\section{Introduction}

Stroke is a main cause of mortality [1] and the leading cause of adult long-term disability in industrialized countries [2]. Despite the decreasing incidence rate, better survival rates are expected to lead to increased prevalence rate and the need for efficient rehabilitation and healthcare policies for patients with stroke [3].

In describing activities of daily living (ADL) as a measure of long-term outcome of stroke, it is important to distinguish between basic ADL (BADL) and instrumental ADL (IADL) [4]. BADL are self-maintenance skills, such as bathing, dressing, and toileting. In this study, IADL refer to more complex activities, including

Received: 10 January 2020, Accepted: 16 September 2020

Corresponding to: Kohei Kusuda, Kyoto Min-Iren Asukai Hospital, Kyoto, Kyoto Japan

e-mail: ten5peace@yahoo.co.jp

(C2021 Japanese Association of Occupational Therapists domestic chores, social activities, and gainful work [5]. Because IADL are activities that typically must be performed by the stroke survivor to continue living in the community [6], it will be difficult to predict whether patients can resume IADL or domestic chores while they are hospitalized. Among IADL, "domestic chores" is the area where occupational therapy support is needed for independent living [7]. Walsh et al. [8] found that two-thirds of stroke survivors need help with domestic chores up to five years after stroke. Besides, domestic chores are affected by various factors such as mobility, cognitive function, family support, and lifestyle [9], and stroke patients may be unable to resume the domestic chores that they performed before.

The International Classification of Functioning, Disability, and Health (ICF) defined preparing meals and doing housework as domestic chores [10]. Studies in Japan have defined preparing meals, cleaning, washing clothes, and shopping as items of domestic chores because these activities support living [11]. According 
to the Statistics Bureau of Japan 2017 [12], these are the following characteristics regarding the implementation time for housework among Japanese individuals; women spend more time doing housework than men in all ages; women spend less time doing housework after 65 years of age, while men spend more time doing housework after 65 years of age; people living with a partner spend more time doing housework than people living alone.

There are two types of IADL assessment tool. One assesses the patient's ability to perform IADL, e.g., the Lawton scale, and the other assesses the frequency at which IADL is performed, e.g., the Frenchay Activities Index (FAI). We chose FAI as the assessment tool for IADL and domestic chores as it assesses the frequency at which patients perform IADL, which provides a motr correct reflection of daily living than the tool that assesses the patient's ability to perform IADL.

IADL generally require higher physical functions, such as walking abilities $[3,9,13]$, balance abilities [13], cognitive functions such as memory [14], executive function $[9,15]$, and psychological function such as self-efficacy $[13,16]$. Conversely, IADL is negatively affected by paralyzed limb function [17], cognitive dysfunction [15], and depression [15, 18]. As stated, while several studies analyzed factors that affect IADL after stroke, only few studies evaluated the factors affecting domestic chores after stroke. Moreover, these studies use tools that assess the patient's ability to perform IADL, and few studies use the tools that assess the frequency at which patients perform IADL. Therefore, this study aimed to clarify the factors that affect frequency of doing domestic chores after stroke. In this study, domestic chores include preparing meals, washing dishes, washing clothes, cleaning, and shopping.

\section{Materials and Methods}

\subsection{Participants}

The dataset utilized in this study was acquired between 2015 and 2018 from the rehabilitation ward. All participants were patients with first-time stroke (cerebral infraction, cerebral hemorrhage, or subarachnoid hemorrhage). Other inclusion criteria were as follows: First, the patients were independent in walking in the hospital. In the current study, this was defined by an FIM score of 6 or 7 in the walking domain, indicating that participants were included if they used a stick or orthosis. Second, they had been independent in domestic chores before stroke onset. In the current study, this was defined by FAI score of 15 in the domestic chores domain, indicating that they had usually done preparing meals, washing dishes, washing clothes, cleaning, and shopping before stroke onset. Patients were excluded if they had severe hyper-brain dysfunction, such as aphasia, apraxia, and unilateral spatial neglect, or other serious conditions other than stroke that could affect the study outcome.

All participants underwent a 60-180-min supervised rehabilitation program daily while they were in the rehabilitation ward. The program consisted of physical and occupational therapies for all participants and speech therapy if needed. In addition to individual rehabilitation, they underwent walking practice that was tailored to individual abilities with a nurse or caregiver. After hospital discharge, they used a home help service or rehabilitation as needed.

\subsection{Procedure}

The researcher approached individuals who met the inclusion criteria during hospitalization and we included them as the participants. A written informed consent was obtained from the participants. Demographic data and clinical characteristics were collected from the medical records of our hospital. Other outcome measures except for post-stroke FAI, including the subscale of domestic chores, were assessed during the hospitalization. The assessment time of these outcome measures were the final assessment before they were discharged from the hospital. Pre-stroke FAI and subscale of domestic chores were assessed by retrospectively recalling the performance of domestic chores and other IADL before stroke onset. Post-stroke FAI and subscale of domestic chores were assessed by face-to-face interviews at their residence post discharge (1 month) from the hospital. Ethical approval was provided by the researchers' host university and written consent was obtained from all participants.

\subsection{Outcome measures}

\subsubsection{Functioning}

\subsubsection{Basic activities of daily living}

BADL was assessed using the Functional Independence Measure (FIM) [19]. FIM is an 18-item, 7-level scale that was developed to assess the functional independence of patients with neurological impairments. FIM consists of the motor domain (FIM-M) and cognitive domain (FIM-C). FIM-M includes 13 items related to $\mathrm{ADL}$, and the total score is 91 points. Moreover, FIM-C includes 5 items associated with communication and socialization.

\subsubsection{Instrumental activities of daily living}

IADL was measured using FAI [20]. It comprises 15 items (preparing meals, washing dishes and clothes, cleaning, local shopping, heavy housework, social outings, walking outdoors, active hobby, driving a car/ traveling on bus, outing/car rides, gardening, household 
and/or car maintenance, reading books, gainful work), and each item was scored from 0 to 3 . In the FAI, the frequency of each activity is assessed; the higher the frequency of the item, the higher is the score. The FAI consists of a single summary score, ranging from 0 (inactive) to 45 (highly active). Previous studies showed that the FAI is a valid, reliable, and sensitive measure of social activity and IADL in patients with stroke [21, 22]. The FAI was developed to provide information on the level of activities observed before and after stroke [21]. Pre- and post-stroke FAI were assessed to show the levels of premorbid and post-stroke activities. Prestroke FAI and both pre- and post- stroke performance of domestic chores in the FAI were used as variables.

\subsubsection{Domestic chores}

In this study, we defined preparing meals, washing dishes, washing clothes, cleaning, and local shopping as domestic chores in the items of the FAI. Preparing meals and washing clothes are scored three points if performed most days in a week, and the other items are scored three points if performed at least weekly.

\subsubsection{Demographic data and clinical characteristics of stroke}

Information on sex, age, and number of family members was obtained as demographic data. For clinical characteristics of stroke, data on the etiology, stroke type, and affected hemisphere were collected.

\subsubsection{Physical function}

Balance ability was assessed using the Berg Balance Scale (BBS) and Timed Up and Go (TUG) test. The BBS is a 14-item measure of balance and risk for falls in older adults through direct observation on a scale of 0 (inability to complete the task) to 4 (independent task completion) [23]. The TUG test was developed to evaluate functional mobility of frail elderly individuals and measure mobility speed [24]. The participants sat down in a chair and then were timed with a stopwatch to determine how quickly they could stand up and walk $3 \mathrm{~m}$, turn a corner, walk back, and sit down again. The severity of paralysis was assessed using the Stroke Impairment Assessment Set - motor function (SIAS-M). The SIAS includes 22 items based on nine types of dysfunction. Each item could be scored as 3 or 5 points. SIAS-M includes 5 items that assess abilities of affected arms and legs, with a total score 25 points.

\subsubsection{Cognitive function}

Attention was assessed using the Trail Making Test parts A and B (TMT-A and TMT-B, respectively) and WAIS-III subtest of the symbol digit substitution test
(SDMT). In TMT-A, the respondent is instructed to connect randomly arranged circles containing numbers from 1 to 25 following the number sequence and perform it as quickly as possible. The task in TMT-B is similar to that in TMT-A, but the respondent has to alternate numbers and letters. In the SDMT, a coding key showed nine abstract symbols, each paired with a number. Below the key, a series of symbols were presented, and the participants were asked to write down the corresponding numbers as quickly as possible. The number of correct substitutions in a 120-s interval was used as the score. Executive function was assessed using the Tower of Hanoi (TOH) and Behavioral Assessment of the Dysexecutive Syndrome Dysexecutive Questionnaire (BADSDEX). The TOH consists of a wooden structure with a rectangular base with three evenly spaced pegs and several wooden discs. BADS-DEX includes a 20-item questionnaire on executive-type behavioral problems. Each item is scored on a scale of 0 (not at all) to 4 (always), and the maximum score is 80 points. The higher the score, the stronger the trend of dysexecutive function. Memory was assessed using the Rivermead Behavioral Memory Test (RBMT). The RBMT is designed to tap the participant's memory in performing daily tasks. The RBMT assesses different types of memory, such as associative memory, prospective memory, visual memory, verbal memory, topographic memory, control, and recognition strategies [25]. RBMT produces a global score from 0 to 24 points.

\subsubsection{Psychological function}

Depression was assessed using the self-rated, 15item, Geriatric Depression Scale (GDS). The GDS includes 15 items on depression, asked over several weeks. Subjects answer each question with "Yes" or "No," and scores on the GDS range from 0 to 15 . A score $<5$ indicates no depression, a score from 5 to 10 indicates suspected depression, and a score $>10$ indicates probable depression. The GDS is a reliable and valid selfrating depression screening scale for older individuals and stroke survivors [26]. Self-efficacy was assessed using the 13-item Stroke Self-Efficacy Questionnaire (SSEQ). SSEQ was developed by Jones et al. [27]. This self-report questionnaire comprises 13 items regarding common functional tasks (e.g., getting comfortable in bed, walking, and dressing) and self-management (e.g., coping with frustration of the consequences of stroke). Subjects answer each question between 0 (not at all confident) and 10 (extremely confident), and total scores range from 0 to 130 . The SSEQ has face validity, excellent internal consistency (Cronbach alpha, .90), and criterion validity with the Falls Efficacy Scale [28] (Table 1). 
Table 1 List of outcome measures

\begin{tabular}{lll}
\hline Demographics & \multicolumn{1}{c}{ Age, Sex, Family } \\
\hline Clinical characteristics & \multicolumn{1}{c}{ Stroke type, Affected hemisphere, } \\
\hline \multirow{2}{*}{ Physical function } & severity of paralysis & Stroke Impairment Assessment Set (SIAS-M) \\
\cline { 2 - 3 } & balance ability & Berg Balance Scale (BBS), Timed up and Go Test (TUG) \\
\cline { 2 - 3 } Cognitive function & executive function & $\begin{array}{l}\text { Trail Making Test A and B (TMT-A and TMT-B) } \\
\text { Symbol Digit Substitution Test (SDMT) }\end{array}$ \\
\cline { 2 - 3 } & Dysexecutive Syndrome Dysexecutive Questionnaire (BADS-DEX) \\
\cline { 2 - 3 } Pshychological function & depression & Revermed Behaviral Memory Test (RBMT) \\
\cline { 2 - 3 } & self efficacy & Seriatric Depression Scale-15 (GDS-15) \\
\hline \multirow{2}{*}{ Functioning } & ADL & Functional Independence Measure (FIM) \\
\cline { 2 - 3 } & IADL & Frenchay Activities Index (FAI) \\
\hline
\end{tabular}

a; collected from the medical records of our hospital, b; assessed during the participants were in the hospital, c; Pre-stroke FAI was assessed by retrospectively recalling the performance before stroke onset. Post-stroke FAI was assessed by face-to-face interviews in their home 1 month after discharge from the hospital.

\subsection{Statistical analyses}

All analyses of data were conducted using R 3-5-0 software. A significance level of .05 was established.

Descriptive statistics were used to describe the demographic data of age, sex, and family. The stroke characteristics included stroke type, affected hemisphere, score in the National Institute of Health Stroke Scale (NIHSS) at onset, assessment time from onset, functioning, and other outcomes. The post-stroke FAI score of each item of the domestic chores domain was presented. To investigate the relationship between post-stroke scores of domestic chores domain and other variables, the Spearman's rank-order correlation was used.

\section{Results}

Twenty-nine participants were enrolled in this study. The mean age of participants was $72.5 \pm 7.8$ years, ranging from 57 to 86 years. Seven participants (24.1\%) were male. Moreover, 16 participants $(55.2 \%)$ had cerebral infarction, 10 (34.5\%) had cerebral hemorrhage, and $3(10.3 \%)$ had subarachnoid hemorrhage. The right hemisphere was affected in 12 participants (41.4\%), while the left hemisphere was affected in 16 participants $(55.2 \%)$. The median NIHSS score at stroke onset was 5.5 (3-11.3), and the FIM summary score upon hospital discharge was 121 (117-123). This indicated that participants were mild to moderate stroke survivors, and their independence rate of ADL was relatively high (Table 2).

The proportion of participants rating themselves at the highest level of actual participation was $58.6 \%$ for
Table 2 Description of demographic data, stroke characteristics and pre- and post- stroke FAI summary score of participants $(n=$ 29)

\begin{tabular}{lr}
\hline Demographic data & \\
Age (years) & $72.5 \pm 7.8$ \\
Sex - Male (n) & $7(24.1 \%)$ \\
Family - Living alone (n) & $22(75.9 \%)$ \\
Stroke characteristics & \\
Stroke type - Cerebral Infarction (n) & $16(55.2 \%)$ \\
$\quad$ - Cerebral Hemorrhage (n) & $10(34.5 \%)$ \\
$\quad$ - Subarachnoid Hemorrhage (n) & $3(10.3 \%)$ \\
Affected hemisphere - Right (n) $\quad 12(41.4 \%)$ \\
$\quad$ - Left (n) & $16(55.2 \%)$ \\
$\quad$ Middle (n) & $1(3.4 \%)$ \\
NIHSS score at stroke onset & $5.5(3-11.3)$ \\
Assessment time from stroke onset (days) & $121.6 \pm 62.3$ \\
Functioning & \\
FIM summary score at discharge from the hospital & $121(117-123)$ \\
FIM-M score at discharege from the hospital & $88(85-90)$ \\
FIM-C score at discharge from the hospital & $33(31-35)$ \\
pre-stroke FAI summary score & $32(29-34.3)$ \\
post-stroke FAI summary score & $23(15.5-27)$ \\
\hline
\end{tabular}

Values given as mean $\pm \mathrm{SD}$ for ratio scale and median $(\mathrm{Q} 1-\mathrm{Q} 3)$ for ordinal scale and $\mathrm{n}(\%)$ for normal data NIHSS, National Institute of Health Stroke Scale; FIM, Functional Independence Measure; FIM-M, FIM motor domain; FIM-C, FIM cognitive domain; FAI, Frenchay Activities Index

preparing meals, $65.5 \%$ for local shopping, $69.0 \%$ for cleaning, and $82.8 \%$ for washing dishes and clothes. In contrast, the proportion of participants who never participated in these activities was $27.6 \%$ for preparing meals, $24.1 \%$ for cleaning and local shopping, and $13.8 \%$ for 
Table 3 The post-stroke score of each item of the domestic chores domain of the FAI $(n=29)$

\begin{tabular}{lcccc}
\hline & \multicolumn{4}{c}{$\begin{array}{c}\text { Median (Q1-Q3): 13 (9-15) } \\
\text { min-max: 0-15 }\end{array}$} \\
\cline { 2 - 5 } & Never & Under once weekly & $1-2$ times a week & Most days \\
\hline Preparing meals & $8(27.6 \%)$ & $2(6.9 \%)$ & $2(6.9 \%)$ & $17(58.6 \%)$ \\
Washing dishes & $4(13.8 \%)$ & $0(0.0 \%)$ & $1(3.4 \%)$ & $24(82.8 \%)$ \\
\hline & Never & $1-2$ times in 3months & $3-12$ times in 3months & At least weekly \\
\hline Washing clothes & $4(13.8 \%)$ & $1(3.4 \%)$ & $0(0.0 \%)$ & $24(82.8 \%)$ \\
Cleaning & $7(24.1 \%)$ & $2(6.9 \%)$ & $0(0.0 \%)$ & $20(69.0 \%)$ \\
Local Shopping & $7(24.1 \%)$ & $2(6.9 \%)$ & $1(3.4 \%)$ & $19(65.5 \%)$ \\
\hline
\end{tabular}

Values given as $\mathrm{n}(\%)$

washing dishes and clothes (Table 3).

The average SIAS-M score was $22.9 \pm 4.2$. The FIM-M score was $86.9 \pm 3.9$, and pre-stroke FAI score was $31.5 \pm 3.7$. The TUG test $(r=0.41, p=0.03)$, SSES $(r=0.54, p<0.01)$, and FIM-M $(r=0.57, p<0.01)$ results were significantly associated with the domestic chores domain of the FAI. The SIAS-M $(r=0.36, p=$ $0.06)$, TMT-B $(r=-0.34, p=0.07)$, TOH $(r=0.35$, $p=0.08)$, BADS-DEX $(r=-0.37, p=0.07)$, and RBMT $(r=0.37, p=0.06)$ scores had a mild but insignificant association the domain $(|r|>0.3)$. Other outcome measures were not significantly associated with the domestic chores domain of the FAI (Table 4). Of the 29 participants, two were missing data for TUG, one for SDMT, three for ToH, four for BADS-dex, three for RBMT, one for GDS-15, and one for SSEQ.

\section{Discussion}

The current study investigated post-stroke frequency of domestic chores using the FAI. By using a criterion of pre-stroke score of domestic chores domains on FAI was the maximum. We also investigated the participant's demographics, clinical characteristics, physical function, cognitive function, psychological function, and pre- and post-stroke functioning and their relationship to the frequency of post-stroke domestic chores. The strengths of our study are as follows: First, because our participants had been independent of domestic chores before stroke onset, we could analyze the impact of stroke on domestic chores purely. Second, it is easy, accurate, or precise to consider the influence of each factor by focusing on "domestic chores" in various IADL.

Blomgren et al. [4] investigated IADL in 237 young and middle-aged stroke survivors using FAI, with the proportion of participants rating themselves at the highest level of actual participation from a low of $43.9 \%$ for preparing meals to a high of $81.9 \%$ for local shopping. Our sample shows a similar trend for preparing meals.
This implies that preparing meals was the most difficult to resume for patients with stroke in the domestic chores domain. This is because preparing meals is a complex activity that needs high cognitive functions, such as executive function [14] and practical balance ability, compared to washing dishes, washing clothes, and cleaning. Clearly, preparing meals is important for independent living [29] and an individual's sense of life satisfaction [30]. In contrast, washing dishes and washing clothes were activities that can be resumed after stroke. This implies that interventions for washing dishes and washing clothes are relatively easy to improve the independence of domestic chores after stroke.

The median FIM-M score of our sample was 88 , but pre- and post-stroke median FAI scores were 32 and 23 , respectively. This indicates that IADL can decrease despite full independence in BADL, as measured by the FIM. In a previous study, Edwards et al. [31] investigated stroke-related symptoms in patients with mild stroke. The results showed that, although $95 \%$ of participants had FIM scores of 110 , approximately $40 \%$ and $35 \%$ reported problems on social integration and household management, respectively. There are several studies that showed similar results on ADL and IADL of patients with stroke [32]. Despite the difference in the assessment tools between this and previous studies, our results highlight the importance of assessment of IADL in patients with stroke, particularly those who can perform ADL independently.

The results of this study showed that the TUG test $(r=-0.41, p<0.01)$, SSEQ $(r=0.54, p<0.01)$, and FIM-M $(r=0.57, p<0.01)$ scores were significantly associated with frequency of domestic chores. The TUG test evaluates gait and balance performance [33]. Because the TUG test can be performed easily, it can be used as a screening tool for reflecting domestic chores after stroke. The concept of self-efficacy is described as the confidence in one's ability to perform a task or specific behavior. Thus, the strongest way of influencing 
Table 4 Descriptive statisitics and spearman's correlation with the domain of domestic chores of FAI after stroke $(n=29)$

\begin{tabular}{|c|c|c|c|c|}
\hline & $\mathrm{n}$ & average or Median & Spearman's Correlation & $p$-value \\
\hline \multicolumn{5}{|l|}{ Demographics } \\
\hline sex - Male & 29 & $7(24.1 \%)$ & 0 & 1 \\
\hline age & 29 & $72.5(7.8)$ & -0.13 & 0.49 \\
\hline family - Living Alone & 29 & $22(75.9 \%)$ & -0.23 & 0.22 \\
\hline \multicolumn{5}{|l|}{ Physical function } \\
\hline SIAS-M (min-max: 0-25) & 29 & $25(23-25)$ & 0.36 & 0.06 \\
\hline BBS (min-max: 0-56) & 29 & $53(50-55)$ & 0.29 & 0.13 \\
\hline TUG (second) & 27 & $11.9(8.4)$ & -0.41 & $0.03 *$ \\
\hline \multicolumn{5}{|l|}{$\begin{array}{l}\text { Cognitive function } \\
\text { attention }\end{array}$} \\
\hline TMT-A (second) & 29 & $193.3(126.8)$ & -0.22 & 0.26 \\
\hline TMT-B (second) & 29 & $239.1(129.8)$ & -0.34 & 0.07 \\
\hline $\begin{array}{l}\text { SDMT (min-max: 0-133) } \\
\text { executive function }\end{array}$ & 28 & $39(32-51)$ & 0.3 & 0.12 \\
\hline ToH (min-max: 0-9) & 26 & $4.5(2-6)$ & 0.35 & 0.08 \\
\hline $\begin{array}{l}\text { BADS-DEX (min-max: 0-80) } \\
\text { memory }\end{array}$ & 25 & $17(9-24)$ & -0.37 & 0.07 \\
\hline RBMT (min-max: 0-24) & 26 & $18(15-20)$ & 0.37 & 0.06 \\
\hline \multicolumn{5}{|l|}{ Psychological function } \\
\hline GDS-15 (min-max: 0-15) & 28 & $5(3-8)$ & -0.07 & 0.73 \\
\hline SSEQ (min-max: 0-130) & 28 & $105(83.3-117)$ & 0.54 & $<0.01 *$ \\
\hline \multicolumn{5}{|l|}{ Functioning } \\
\hline FIM-M (min-max: 13-91) & 29 & $88(85-90)$ & 0.57 & $<0.01 *$ \\
\hline pre-stroke FAI (min-max: 0-45) & 29 & $32(29-34.3)$ & 0.21 & 0.28 \\
\hline
\end{tabular}

${ }^{*} p<0.05$. Values given as mean (SD) for ratio scale and median (Q1-Q3) for ordinal scale and $\mathrm{n}(\%)$ for normal data

SIAS-M, the Stroke Impairment Assessment Set Motor function; BBS, the Berg Balance Scale; TUG, the Timed Up and Go test; TMT-A and TMT-B, the Trail-Making Test part A and B; SDMT, the symbol digit substitution test; ToH, the Tower of Hanoi; BADS-DEX, the Behavioral Assessment of the Dysexecutive Syndrome Dysexecutive Questionnaire; RBMT, the Rivermead Behavioral Memory Test; GDS-15, the self-rated 15-item Geriatric Depression Scale; SSEQ, the 13-item Stroke Self-Efficacy Questionnaire; FIM-M, the Functional Independence Measure Motor function; FAI, the Frenchay Activities Index

self-efficacy is a mastery experience through successful performance of a task [34]. These results imply that patients with stroke should improve not only physical or cognitive function but also self-efficacy to resume domestic chores after stroke. While participants of this study had a high independence rate of BADL with a high FIM-M score, it is still significantly correlated with domestic chores. This suggests that ADL is the basis of domestic chores, and it is important to evaluate BADL to reflect IADL.

Blomgren et al. showed that stroke survivors who are older, men, and living with a partner report low frequencies of performing IADL using FAI. However, in our study, age, sex, and living alone were not associated with domestic chores. This is possibly because participants of the current study had been independent in domestic chores before the onset of stroke compared to those in other studies. Adjustment for pre-stroke functioning should provide a better insight into possible mechanisms for such age and sex differences [35]. Unlike previous studies, cognitive function did not show a statistical relationship with domestic chores after stroke. Our study found similar trends with those of previous studies [36] that executive function or memory, rather than attention, with the more complex tests (TMT-B rather than TMT-A) had associations with domestic chores, while there were no significant associations between cognitive functions and domestic chores. In previous studies that examined the patient's ability to perform IADL, cognitive assessments were performed in the acute phase $[14,15]$ and the pre-morbid performance of IADL was not considered [14-16]. In the current study, cognitive assessments were performed in the subacute phase where the pre-morbid frequency at which patients performed IADL was considered in participants who were mild to moderate stroke patients and had been independent of domestic chores before the stroke. For these reasons, the cognitive function of our participants 
did not affect the frequency of domestic chores strongly.

While most previous studies that examined the patient's ability to perform IADL showed a significant relationship between post-stroke depression and functioning $[15,18]$, depression was not related to the frequency at which patients performed domestic chores in this study. LeBrasseur et al [18] demonstrated that the GDS score of chronic stroke participants living in the community was significantly associated with the management role domain (the organization and management of socially) and without the instrumental role domain (the ability to perform activities in the home and in the community). The instrumental role domain in their study is similar to the role of domestic chores in our study. Unlike previous studies, assessment time of depression and performance of domestic chores was slightly different in our study. Besides, our samples showed relatively low median GDS score of 5 points and the GDS is a dichotomous measure and was not sufficiently sensitive. Moreover, we investigated the frequency at which patients perform domestic chores, while previous studies have investigated the patient's ability to perform domestic chores. As stated, it is possible that depression was not related to domestic chores in this study.

\subsection{Study limitations}

The current study has several potential limitations. Samples were included if they were independent of walking after stroke and did not have severe hyper-brain dysfunction. These inclusion criteria resulted in selection of only patients with mild stroke (FIM-M median score, 85) without severe hyper-brain dysfunction. The sample size was relatively small, and the term from the stroke onset was not consistent. Given the small sample size, the results from this study provide a preliminary overview of the relationship between the different possible factors influencing the frequency at which patients perform domestic chores. Besides the small sample size made it difficult to use predictive or multivariate models for assessing the relationship between the possible factors for domestic chores and predictive power of those factors.

Future studies should include more participants and use predictive or multivariate models to look for factors predicting the frequency at which patients perform domestic chores after stroke. Besides, future studies should collect acute, sub-acute, and chronic phase data of stroke patients and conduct longitudinal research to improve the prediction accuracy.

\subsection{Conclusion}

We found that balance ability, basic ADL, and self-efficacy were statistically associated with post- stroke frequency of domestic chores. Our study extends these findings to include the domestic chores domain. Our results imply that stroke patients should improve not only physical or cognitive function but also selfefficacy to resume domestic chores.

\section{Acknowledgements}

We would like to thank all participants of the present study. We would like to thank Editage (www.editage. com) for English language editing.

\section{References}

[1] D'Alessandro G, Gallo F, Vitaliano A, Del Col P, Gorraz F, De Cristofaro R, et al. Prevalence of stroke and stroke-related disability in Valle d'Aosta, Italy. Neurol Sci. 2010; 31: $137-41$.

[2] Verheyden G, Nieuwboer A, Feys H, Thijs V, Vaes K, De Weerdt W. Discriminant ability of the Trunk Impairment Scale: A comparison between stroke patients and healthy individuals. Disabil Rehabil. 2005; 27: 1023-8.

[3] Singam A, Ytterberg C, Tham K, von Koch L. Participation in Complex and Social Everyday Activities Six Years after Stroke: Predictors for Return to Pre-Stroke Level. PLoS One. 2015; 10: e0144344.

[4] Blomgren C, Jood K, Jern C, Holmegaard L, Redfors P, Blomstrand $\mathrm{C}$, et al. Long-term performance of instrumental activities of daily living (IADL) in young and middle-aged stroke survivors: Results from SAHLSIS outcome. Scand J Occup Ther. 2018; 25: 119-26.

[5] Blomgren C, Samuelsson H, Blomstrand C, Jern C, Jood $\mathrm{K}$, Claesson L. Long-term performance of instrumental activities of daily living in young and middle-aged stroke survivors-Impact of cognitive dysfunction, emotional problems and fatigue. PLoS One. 2019; 14: e0216822.

[6] Carod-Artal FJ, González-Gutiérrez JL, Herrero JA, Horan T, De Seijas EV. Functional recovery and instrumental activities of daily living: follow-up 1-year after treatment in a stroke unit. Brain Inj. 2002; 16: 207-16.

[7] Inomata E, Kobayashi N. An exploratory study of solutions for certified Long-Term Care elderly living alone: A focus on household tasks (in Japanese). Jpn Occup Ther Res (sagyouryouhou). 2014; 33: 230-40.

[8] Walsh ME, Galvin R, Loughnane C, Macey C, Horgan NF. Community re-integration and long-term need in the first five years after stroke: results from a national survey. Disabil Rehabil. 2015; 37: 1834-8.

[9] Kusuda K, Tanemura R, Saito Y. The predictor of IADL abilities of stroke patients living at home: Frequency change of household activities after stroke (in Japanese). Jpn Occup Ther Res (sagyouryouhou). 2018; 37: 128-36.

[10] World Health Organization. The International Classification of Functioning, Disability and Health [online]. 2001 [cited 2020 June 5]. Available from: https:/www.who.int/ classifications/icf/en/. 
[11] Miyake N, Yanagihara K, Shindo N. House work of female stroke patients (in Japanese). Jpn J Rehabil Med (rihabiriteishonigaku). 1999; 36: 644-8.

[12] Statistics Bureau of Japan. Results of the Basic Survey on Social Life 2016 (in Japanese) [online]. 2017 [cited 2020 June 5]. Available from: https://www.stat.go.jp/data/ shakai/2016/kekka.html.

[13] Schmid AA, Van Puymbroeck M, Altenburger PA, Dierks TA, Miller KK, Damush TM, et al. Balance and balance self-efficacy are associated with activity and participation after stroke: a cross-sectional study in people with chronic stroke. Arch Phys Med Rehabil. 2012; 93: 1101-7.

[14] Yantz CL, Johnson-Greene D, Higginson C, Emmerson L. Functional cooking skills and neuropsychological functioning in patients with stroke: an ecological validity study. Neuropsychol Rehabil. 2010; 20: 725-38.

[15] Babulal GM, Huskey TN, Roe CM, Goette SA, Connor LT. Cognitive impairments and mood disruptions negatively impact instrumental activities of daily living performance in the first three months after a first stroke. Top Stroke Rehabil. 2015; 22: 144-51.

[16] Frost Y, Weingarden H, Zeilig G, Nota A, Rand D. SelfCare Self-Efficacy Correlates with Independence in Basic Activities of Daily Living in Individuals with Chronic Stroke. J Stroke Cerebrovasc Dis. 2015; 24: 1649-55.

[17] Andrenelli E, Ippoliti E, Coccia M, Millevolte M, Cicconi B, Latini L, et al. Features and predictors of activity limitations and participation restriction 2 years after intensive rehabilitation following first-ever stroke. Eur $\mathrm{J}$ Phys Rehabil Med. 2015; 51: 575-85.

[18] LeBrasseur NK, Sayers SP, Ouellette MM, Fielding RA. Muscle impairments and behavioral factors mediate functional limitations and disability following stroke. Phys Ther. 2006; 86: 1342-50.

[19] Granger CV. The emerging science of functional assessment: our tool for outcomes analysis. Arch Phys Med Rehabil. 1998; 79: 235-40.

[20] Holbrook M, Skilbeck CE. An activities index for use with stroke patients. Age Ageing. 1983; 12: 166-70.

[21] Wade DT, Legh-Smith J, Langton Hewer R. Social activities after stroke: measurement and natural history using the Frenchay Activities Index. Int Rehabil Med. 1985; 7: 176-81.

[22] Lin KC, Chen HF, Wu CY, Yu TY, Ouyang P. Multidimensional Rasch validation of the Frenchay Activities Index in stroke patients receiving rehabilitation. J Rehabil Med. 2012; 44: 58-64.

[23] Berg K, Wood-Dauphinee S, Williams JI. The Balance Scale: reliability assessment with elderly residents and patients with an acute stroke. Scand J Rehabil Med. 1995; 27: 27-36.

[24] Podsiadlo D, Richardson S. The timed "Up \& Go": a test of basic functional mobility for frail elderly persons. J Am Geriatr Soc. 1991; 39: 142-8.

[25] Requena C, Turrero A, Ortiz T. Six-Year Training Improves Everyday Memory in Healthy Older People. Randomized Controlled Trial. Front Aging Neurosci. 2016; 8: 135.

[26] Nannetti L, Paci M, Pasquini J, Lombardi B, Taiti PG. Motor and functional recovery in patients with poststroke depression. Disabil Rehabil. 2005; 27: 170-5.

[27] Jones F, Partridge C, Reid F. The Stroke Self-Efficacy Questionnaire: measuring individual confidence in functional performance after stroke. J Clin Nurs. 2008; 17: 244-52.

[28] Tinetti ME, Mendes de Leon CF, Doucette JT, Baker DI. Fear of falling and fall-related efficacy in relationship to functioning among community-living elders. J Gerontol. 1994; 49: M140-7.

[29] Poole J, Sadek J, Haaland K. Meal Preparation Abilities After Left or Right Hemisphere Stroke. Arch Phys Med Rehabil. 2011; 92: 590-6.

[30] Johnston MV, Goverover Y, Dijkers M. Community activities and individuals' satisfaction with them: quality of life in the first year after traumatic brain injury. Arch Phys Med Rehabil. 2005; 86: 735-45.

[31] Edwards DF, Hahn M, Baum C, Dromerick AW. The impact of mild stroke on meaningful activity and life satisfaction. J Stroke Cerebrovasc Dis. 2006; 15: 151-7.

[32] Mayo NE, Wood-Dauphinee S, Côté R, Durcan L, Carlton J. Activity, participation, and quality of life 6 months poststroke. Arch Phys Med Rehabil. 2002; 83: 1035-42.

[33] Sota K, Uchiyama Y, Ochi M, Matsumoto S, Hachisuka K, Domen K. Examination of Factors Related to the Effect of Improving Gait Speed With Functional Electrical Stimulation Intervention for Stroke Patients. PM R. 2018; 10: 798-805.

[34] Bandura A. Self-efficacy. In Encyclopedia of Human Behavior Vol. 4. New York: Academic Press; 1994.

[35] Gall SL, Tran PL, Martin K, Blizzard L, Srikanth V. Sex differences in long-term outcomes after stroke: functional outcomes, handicap, and quality of life. Stroke. 2012; 43: 1982-7.

[36] Cahn-Weiner DA, Farias ST, Julian L, Harvey DJ, Kramer JH, Reed BR, et al. Cognitive and neuroimaging predictors of instrumental activities of daily living. J Int Neuropsychol Soc. 2007; 13: 747-57. 